\title{
Research utilization barriers for emergency medical technicians in Saudi Arabia
}

This article was published in the following Dove Press journal:

Advances in Medical Education and Practice

\section{Osama A Samarkandi \\ Adel S Bashatah ${ }^{2}$ \\ Anas A Khan ${ }^{3}$ \\ Abdulmajeed $M$ Almobrad ${ }^{4}$ \\ Bronwyn Beovich ${ }^{5}$ \\ Brett Williams $s^{5,6}$}

'Basic Science Department, eLearning and IT Unit, Prince Sultan College for Emergency Medical Services, King Saud University, Riyadh, Saudi Arabia; ${ }^{2}$ College of Nursing, King Saud University, Riyadh, Saudi Arabia; ${ }^{3}$ Emergency Medicine Department, College of Medicine, University Medical City, King Saud University, Riyadh, Saudi Arabia; ${ }^{4}$ EMS Department, Prince Sultan College for EMS, King Saud University, Riyadh, Saudi Arabia; ${ }^{5}$ Department of Community Emergency Health and Paramedic Practice, Faculty of Medicine, Nursing and Health Sciences, Monash University, Melbourne, VIC, Australia; 'Division of Paramedicine, School of Medicine, University of Tasmania, Hobart, TAS, Australia
Correspondence: Brett Williams Department of Community Emergency Health and Paramedic Practice, Faculty of Medicine, Nursing and Health Sciences, Monash University, Level 2, Building H, Peninsula Campus, McMahons Road, Frankston, VIC 3199, Australia Email brett.williams@monash.edu
Introduction: Translation of research findings into clinical practice has potential to improve health care procedures, increase patient safety, and improve patient outcomes. However, low levels of evidence utilization in clinical practice have been widely reported. Anecdotal evidence suggests that this is also the case for emergency medical technicians (EMTs) in Saudi Arabia. This study aimed to examine the barriers to the utilization of research findings within this cohort. Methods: The BARRIERS scale was used to gather data from a convenience sample of EMTs in Saudi Arabia.

Results: The barriers most commonly rated as "great" or "moderate" were "Implications for practice are not made clear", "The relevant literature is not compiled in one place", and "The EMT feels the benefits of changing practice will be minimal". However, when responses were examined at a subscale level, reliability, as measured by Cronbach's $\alpha$, was suboptimal (range $0.20-0.62$ ).

Discussion: No similar study has been conducted within paramedicine to enable direct comparison of our results; however, the top barriers identified in the present study are also highly rated in some previous studies of nurse cohorts. The low reliability measures of the subscales may demonstrate the importance of context specificity when utilizing this scale and that further research is required to develop a reliable and valid tool for use within this cohort.

Conclusion: The top 2 barriers identified indicate that there may be a need for improvement regarding communication of research evidence to Saudi EMTs. For future studies, translation of the BARRIERS scale may be useful. However, as these EMT courses are taught in English, careful consideration of cultural suitability and more subtle interpretation issues could also be appropriate. Once context-specific barriers are identified and examined, they may inform the development of effective strategies to increase the uptake of research evidence into Saudi EMT practice.

Keywords: emergency medical technician, paramedic, Saudi Arabia, research utilization, evidence-based practice

\section{Background}

Decisions regarding optimum patient care should always reflect the best evidence available at the time of intervention or practice. Historically, these decisions were primarily informed by experience or opinion from more knowledgeable individuals. ${ }^{1}$ The twentieth century brought increased access to information through the use of textbooks and professional journals, with technology resulting in a huge growth of health information and evidence available in the past few decades. ${ }^{1}$ However, studies indicate that it takes an average of 17 years for evidence to be integrated into common 
clinical practice. ${ }^{2}$ Although the amount of research addressing this "evidence-practice gap" "3 has increased considerably over the past few decades, the literature demonstrates that the phenomenon is still pervasive throughout health care. ${ }^{4}$

The use of the best available information to inform health care decisions, primarily in the form of research findings combined with clinical expertise, was formalized as "evidence-based medicine" in the 1990s. It can be defined as "the conscientious, explicit, and judicious use of current best evidence in making decisions about the care of individual patients." ${ }^{\circ}$ This concept has since been expanded throughout health care as evidence-based practice (EBP). The literature uses many interrelated terms to describe the phenomena associated with EBP such as knowledge translation, research utilization, guideline uptake, innovation diffusion, and implementation science. ${ }^{4,7}$ For the purposes of this paper, "research utilization" will be the preferred term.

Utilization of high-quality research findings from randomized controlled trials, research synopses, and information syntheses such as meta-analyses have the potential to improve health care procedures, increase patient safety, and improve patient outcomes. ${ }^{8}$ However, the existence of evidence is typically insufficient for it to be transferred into practice. ${ }^{4}$ For example, it has been reported that only $57 \%$ of Australian patients receive evidence- or consensus-based care, ${ }^{9}$ which is similar to an earlier report of $55 \%$ of patients in the USA receiving "recommended care." "For evidence to be utilized, good quality research first needs to be completed, then the results of this research need to be disseminated to relevant individuals and organizations. This new information then needs to be acknowledged, understood, and accepted, all prior to the possibility of effective implementation into practice and subsequent adherence to the new practice..$^{3,11}$ Barriers to this process may be present at any point and can occur at both the organizational and individual levels. ${ }^{12} \mathrm{~A}$ thorough understanding of context-specific barriers should be gained prior to planning any intervention to increase research utilization, as targeted interventions which take into consideration specific barriers appear to be more successful than those which do not. ${ }^{11}$

Previous research has identified barriers related to the individual, which include lack of time, lack of autonomy to modify practice, level of academic qualification, previous involvement in research, as well as attitudes and beliefs about research and EBP. ${ }^{12,13}$ Organizational barriers may include lack of health information systems, politics, bureaucracy, inadequate facilities, noncompilation of relevant information, isolation from colleagues with research knowledge, and colleague dynamics. ${ }^{14-16} \mathrm{~A}$ recent scoping study of barriers in a variety of health care settings reported that the 5 main organizational barriers were: workload, unsupportive staff, lack of resources, lack of authority to change practice, and a resistant workplace culture. ${ }^{17}$

The barriers to research utilization have been studied extensively within the nursing profession. ${ }^{12,18}$ However, there are far fewer studies within other health professions, ${ }^{13}$ including the emerging profession of paramedicine. ${ }^{19}$ Barriers can differ between different health professions, ${ }^{20}$ again highlighting the importance of assessing barriers according to context. ${ }^{11}$ This is particularly important for the paramedic setting, which is often unpredictable and dynamic. Furthermore, this is important as paramedicine is emerging as a fully fledged profession in many countries developing its own EBP. ${ }^{21}$ As paramedicine in Saudi Arabia continues to develop, it is guided by the strategic vision of the Kingdom, which supports enhancement of research skills and outcomes in all scientific areas, especially within the domain of medicine and health care. ${ }^{22,23}$

The Emergency Medical Service (EMS) in Saudi Arabia is operated by the Saudi Red Crescent Authority (SRCA) and is the local provider of emergency prehospital care and transportation. ${ }^{21}$ The workforce consists of emergency medical technicians (EMTs) and paramedics, medical physicians, as well as firefighters and other ambulance employees. ${ }^{24}$ Although prehospital care and paramedicine are not new in Saudi Arabia, there has been considerable improvements in the professional education available over the last decade. ${ }^{21}$ There has been establishment of a 4-year university-based education, with an increasing use of evidence-based curricula. ${ }^{21,25}$ At present, no national curricula standard exist for paramedic education in Saudi Arabia, and thus the precise curricula content for each university has not been mapped. However, it is recognized that the principles of EBP are included in all programs. Although increasingly exposed to an evidence-based education, there are barriers to research utilization that have been identified anecdotally by EMTs in this setting. These include lack of time, inaccessible research reports, and difficulty related to language barriers.

The rationale for this study was to build on this anecdotal evidence by gathering empirical evidence regarding the issue. Once the barriers to research utilization are identified and examined, results may be used to inform the development of strategies and interventions to enhance the utilization of research findings by EMTs in Saudi Arabia. 


\section{Aim/purpose}

The aim of this study was to explore and identify the perceived barriers encountered by EMTs regarding research utilization in Saudi Arabia.

\section{Methodology \\ Design and setting}

This study was of a cross-sectional, descriptive design where a preexisting survey instrument was used to explore perceptions of barriers to research utilization among EMTs in Saudi Arabia. The study examined responses from EMTs who were employed at 15 SRCA stations within Riyadh, the capital city of Saudi Arabia. Three stations from each of the 5 areas in Riyadh, North, South, East, West, and Middle, were involved, as well as EMTs employed in 3 major Riyadh hospitals.

\section{Sampling technique}

The investigators sought to gather responses from a representative sample of EMTs by recruiting widely. A convenience sample of EMTs from 15 SRCA stations and 3 major hospitals and who were currently employed in the EMS field was obtained. The intended sample was approximately 300 EMTs, which was calculated utilizing the G*Power method (Heinrich-Heine-Universität Düsseldorf, Düsseldorf, Germany). ${ }^{26}$

\section{Instrument}

The BARRIERS scale was used to investigate EMT perceptions of barriers to the use of research evidence in clinical practice. The scale was originally developed by assessing these perceptions among practicing nurses in the USA. ${ }^{27}$ The scale items were developed through a process of literature search as well as feedback from nurses, researchers, and a psychometrician. Items were then tested for face and content validity and subsequently pilot tested. The resulting scale initially consisted of 29 items related to barriers to the use of research findings. However, 1 item "the amount of research information is overwhelming" was removed after completion of a factor analysis. ${ }^{27}$

Each of the remaining 28 items is rated on a 4-point Likert scale (1="to no extent," $2=$ ="to a little extent," $3=$ ="to a moderate extent," $4=$ "to a great extent"), and respondents can also choose a 5="no opinion" alternative. These items can be categorized into 4 subscales associated with utilization of research findings; characteristics of the adapter (clinician), the organization (work setting), the innovation (research findings), and the communication of the information (dissemination and accessibility of research findings). These factors are reflective of the main concepts from Rogers ${ }^{28}$ widely acknowledged Diffusion of Innovations model, which in part states that, for an innovation to be successfully disseminated, it must be communicated over time via particular channels, among the members of a social system.

Upon development, Cronbach $\alpha$ values for the 4 subscales in the BARRIERS questionnaire were shown to be $0.80,0.80$, 0.72 , and $0.65,{ }^{27}$ respectively, demonstrating acceptable reliability for the former 3 subscales ${ }^{29}$ and a lower level for the latter. To test the temporal stability of the instrument, 17 subjects answered the questionnaire twice, 1 week apart. Pearson correlations between the 2 data sets ranged from 0.68 to 0.83 , which indicated acceptable stability over this time frame. ${ }^{27}$ The BARRIERS survey also includes an area to list and rate any barriers to research utilization not listed on the survey, as well as ranking of the 3 greatest barriers. The original BARRIERS questionnaire was modified for use in the present study by changing the word "nurse" to "emergency medical technician," to enhance relevance to the sampled cohort.

\section{Procedure}

The data were collected via self-administered paper-based questionnaires, which were given individually to each participant along with a project explanatory statement. The participants submitted the completed questionnaires to the director officer within the station or hospital they were located. After 3 days, the researcher returned to the director's office to collect the completed questionnaires.

\section{Data analysis}

The Statistics Package for Social Sciences (IBM SPSS Statistics v.23, IBM Corporation, Armonk, NY, USA) was used to determine descriptive statistics for both demographic and survey data. The percentage of participants rating each of the 28 items as a "great" or "moderate" barrier was calculated and then placed into rank order. The Kruskal-Wallis test was also used to compare the medians of the 4 subscale factors to qualification level and the number of years of experience as an EMT. Cronbach $\alpha$ coefficients were also calculated for each subscale to examine internal consistency, as a measure of subscale reliability.

For analysis, the 28 items were grouped into 4 subscales as has been previously described. ${ }^{27}$ These subscales were as follows:

- Characteristics of the adopter (The EMT is unaware of the research, The EMT feels the benefits of changing practice will be minimal, The EMT is isolated from knowledgeable colleagues with whom to discuss the research, The EMT 
sees little benefit for self, The EMT does not see the value of research for practice, There is not a documented need to change practice, The EMT is unwilling to change/try new ideas, The EMT does not feel capable of evaluating the quality of the research);

- Characteristics of the organization (The facilities are inadequate for implementation, The EMT does not have time to read research, The EMT does not feel she/he has enough authority to change patient care procedures, The EMT feels results are not generalizable to own setting, Physicians will not cooperate with implementation, Administration will not allow implementation, Other staff are not supportive of implementation, There is insufficient time on the job to implement new ideas);

- Characteristics of the innovation (The research has not been replicated, The EMT is uncertain whether to believe the results of the research, The research has methodological inadequacies, Research reports/articles are not published fast enough, The conclusions drawn from the research are not justified, The literature reports conflicting results);

- Characteristics of the communication (Research reports/ articles are not readily available, Implications for practice are not made clear, Statistical analyses are not understandable, The research is not relevant to the EMT's practice, The relevant literature is not compiled in 1 place, The research is not reported clearly and readably).

\section{Ethical considerations}

Participants were advised they were not obligated to participate in the study, and they could withdraw from the study without penalties or consequences. Consent was implied by the completion and submission of a questionnaire, and data were deidentified by number codes to maintain confidentiality and anonymity of participants. The project was approved by the Institutional Review Board of the College of Medicine, King Saud University.

\section{Results}

Four hundred questionnaires were distributed, with a response rate of $66 \%(n=264)$. All respondents were male with a mean age of 30 years (range 22-46 years). Ninety-six percent were Saudi by nationality, with $92 \%$ of the sample working for the SRCA, while the remainder were employed within a hospital setting. Eighty-seven percent of respondents had EMS diploma qualifications, while approximately $10 \%$ possessed an EMS bachelor-level qualification. The number of years of EMS experience ranged from 1 to 18 years, with a mean of 5 years. Ninety-eight percent worked in a professional clinical capacity, with the minority employed in academic or administrative roles.

The percentage of respondents who judged each item to be a moderate or great barrier was calculated and placed into rank order (Table 1).

The mean (SD) scores for each of the subscales were calculated and are as follows: Adopter 22.7 (4.8), Organization 22.7 (4.7), Innovation 17.2 (4.0), and Communication 16.8 (3.7).

The top 3 items that were rated as moderate or great barriers were; "Implications for practice are not made clear", "The relevant literature is not compiled in one place", and "The EMT feels the benefits of changing practice will be minimal", the former 2 being categorized within the Communication subscale.

The reliability of the 4 subscales, as measured by Cronbach's $\alpha$, was suboptimal. The values for 3 of the subscales were similar (Adopter $\alpha=0.59$, Organization $\alpha=0.60$, Communication $\alpha=0.62$ ), with the score for Innovation considerably lower at $\alpha=0.20$. The 4 scale factors were compared between sample groups using the Kruskal-Wallis test, based on qualification level and the number of years of experience as an EMT; however, no statistically significant differences were found.

\section{Discussion}

This project aimed to examine the barriers to research utilization by EMTs in Saudi Arabia. The use of research evidence to inform EBP has the potential to improve clinical effectiveness, thereby improving patient outcomes. However, the availability of evidence does not automatically lead to transference into practice, with the literature reporting widely on the barriers faced by many health professions on the path to EBP. As paramedicine is a relatively new and developing profession in Saudi Arabia, the timely identification of barriers to the use of research evidence may enhance the clinical application of an expanding body of knowledge.

The present study used a commonly employed scale, the BARRIERS scale, to measure the barriers to research utilization by EMTs in Saudi Arabia. Due to the lack of similar research undertaken within the prehospital setting, there are difficulties with comparison of the present results with other paramedic studies. As the majority of studies using the BARRIERS scale have been undertaken within the nursing profession, comparison with such studies was performed.

Similar research within nursing has been conducted in Middle Eastern countries, where health care and culture may 
Table I Rank order of barriers

\begin{tabular}{|c|c|c|c|}
\hline $\begin{array}{l}\text { Rank } \\
\text { order }\end{array}$ & Item & Subscale & $\begin{array}{l}\% \text { rating item as a great } \\
\text { or moderate barrier }\end{array}$ \\
\hline I & Implications for practice are not made clear & Communication & 63.7 \\
\hline 2 & The relevant literature is not compiled in I place & Communication & 59.6 \\
\hline 3 & The EMT feels the benefits of changing practice will be minimal & Adopter & 59.1 \\
\hline 4 & Research reports/articles are not published fast enough & Innovation & 58.6 \\
\hline 5 & The EMT is uncertain whether to believe the results of the research & Innovation & 58.3 \\
\hline 6 & The EMT is isolated from knowledgeable colleagues with whom to discuss the research & Adopter & 58 \\
\hline 7 & Statistical analyses are not understandable & Communication & 57.2 \\
\hline 8 & The research is not relevant to the EMT's practice & Communication & 57.2 \\
\hline 9 & Other staff are not supportive of implementation & Organization & 56.9 \\
\hline 10 & The research has not been replicated & Innovation & 56.3 \\
\hline II & The research is not reported clearly and readably & Communication & 56 \\
\hline 12 & The EMT does not feel she/he has enough authority to change patient care procedures & Organization & 55.7 \\
\hline 13 & The EMT feels results are not generalizable to own setting & Organization & 55.1 \\
\hline 14 & The EMT sees little benefit for self & Adopter & 55.1 \\
\hline 15 & The EMT does not see the value of research for practice & Adopter & 55.1 \\
\hline 16 & The conclusions drawn from the research are not justified & Innovation & 55 \\
\hline 17 & Physicians will not cooperate with implementation & Organization & 53.8 \\
\hline 18 & The EMT is unwilling to change/try new ideas & Adopter & 53.6 \\
\hline 19 & The EMT is unaware of the research & Adopter & 53 \\
\hline 20 & There is not a documented need to change practice & Adopter & 52.7 \\
\hline 21 & The research has methodological inadequacies & Innovation & 52.5 \\
\hline 22 & There is insufficient time on the job to implement new ideas & Organization & 51.9 \\
\hline 23 & The EMT does not have time to read research & Organization & 51.7 \\
\hline 24 & The literature reports conflicting results & Innovation & 51.5 \\
\hline 25 & The facilities are inadequate for implementation & Organization & 51 \\
\hline 26 & Administration will not allow implementation & Organization & 50.7 \\
\hline 27 & The EMT does not feel capable of evaluating the quality of the research & Adopter & 50.5 \\
\hline 28 & Research reports/articles are not readily available & Communication & 45.4 \\
\hline
\end{tabular}

Abbreviation: EMT, emergency medical technician.

vary considerably from Western countries. ${ }^{31}$ Two nursing studies from Turkey reported the top barrier as being "the facilities are inadequate for implementation." ${ }^{22,33}$ This barrier also featured in the top 3 ranked barriers of other studies..$^{31,34,35}$ The overarching subscale of "Organization" is frequently represented as a main barrier in this Middle Eastern nursing environment. ${ }^{31,33,34}$ In contrast, "Organization" was only represented once in the top 10 barriers for the present cohort. The most frequently perceived barrier subscale in the present study was "Communication," closely followed by "Innovation" (Table 1). Differences between professions and within different cultures may be due to professional differences associated with the "hidden curriculum" ${ }^{36}$ within different countries, professions, and workplaces, but would need further study to clarify explanations.

However other nursing studies demonstrate substantial differences in the perceptions of some barriers compared with the present results. A systematic review of nursing studies using the BARRIERS scale within predominantly Western countries $^{30}$ found that the following 3 items were most commonly cited in the top 10 barriers: "There is insufficient time on the job to implement new ideas," "The nurse does not have time to read research," and "The nurse does not feel she/he has enough authority to change patient care procedures." However, these rankings were not reflected in the EMT cohort where these items ranked 23, 24, and 12, respectively. The inconsistency in various studies would suggest that the concept of barriers to using research evidence within health care clinical practice is complex and is incompletely understood at present.

The calculated reliability for each subscale in this study is lower than many other studies using the BARRIERS scale, which ranges from 0.67 to 0.88 within the literature. ${ }^{27,37-40} \mathrm{In}$ the present study, the survey was not translated, as the EMT courses in Saudi Arabia are taught in English, and therefore it was thought that translation was unnecessary.

The low values of Cronbach $\alpha$ calculated for each subscale, especially for the "Innovation" subscale, demonstrates suboptimal levels of reliability for this cohort. ${ }^{29}$ So although being assessed as suitable for use in many health environments internationally, the scale version used appears to require modification for use within this cohort. ${ }^{35,37,38,40,41}$ 
Within Saudi Arabia, the BARRIERS scale has been demonstrated to be a reliable tool (Cronbach's $\alpha$ scores $>0.7$ ) when used with a cohort of nurses, ${ }^{42}$ which is inconsistent with the results of the present study. However, this 2012 study examined responses from a cohort which included only $5 \%$ of Saudi nurses, whereas the cohort of the present study consisted of $96 \%$ Saudi EMTs. Moreover, $80 \%$ of the surveyed EMTs reported language difficulties as a barrier to evidence uptake which may have adversely influenced their understanding of the study items. Thus, language and comprehension barriers may be related to the low reliability coefficients demonstrated in this study. The survey as used in the present study was not translated into Arabic as the EMT courses in Saudi Arabia are taught in English, so translation was deemed unnecessary. Also, the tool has also shown satisfactory reliability when used in many other countries which do not have English as a first language such as Turkey, Iran, Norway, Taiwan, and Hong Kong..$^{35,37,38,40,41}$ However, in each of these situations, the survey was modified by forward and backward language translation, or wording was changed to improve cultural suitability. Although translation of the survey in future similar studies may be undertaken, consideration should also be given to cultural and interpretation issues.

Nevertheless, responses to individual items still provide valuable information regarding previously unexamined perceptions among Saudi EMTs. The top 3 barriers identified in the present study (Table 1) have been identified as important barriers in previous studies. The top barrier, "Implications for practice are not made clear" was rated as a "great" or "moderate" barrier by $63.7 \%$ of the present cohort. Comparable results of between $59 \%$ and $67 \%$ have been demonstrated by nursing cohorts. ${ }^{16,37,43}$ Similarly, ratings for the next 2 barriers identified "The relevant literature is not compiled in 1 place" (59.6\%) and "The EMT feels the benefits of changing practice will be minimal" (59.1\%) are comparable to some preexisting studies. ${ }^{37,43,44}$

Previous studies have shown that the reliability of the BARRIERS scale shows associated increases with use of "culturally sensitive language." ${ }^{44}$ Therefore, translation from English into Arabic may prove beneficial for future studies in this field. Development of a valid and reliable instrument to examine barriers to research output with the present cohort should also include an assessment of wording/phrasing and context suitability for Saudi culture and Arabic language. Furthermore, it has been suggested that close-ended questions such as used in the BARRIERS tool only evaluate prelabeled barriers, and that the use of more open-ended methodologies may expose further context-specific information not included in the survey. ${ }^{45}$

\section{Limitations}

There are a number of potential limitations for this study. First, although wide convenience sampling was utilized, the cohort obtained may not be representative of the entire Saudi EMT population. Second, answering a self-report questionnaire in a socially desirable manner may result in a response bias, and a response rate of only $66 \%$ may contribute to a nonresponse bias. That is, it is unknown whether the responses of those who chose not to participate would have modified the results. Additionally, it was calculated that a cohort of approximately 300 was required to adequately power the study. The lower actual participant number may limit generalizability of the study. Similarly, the low reliability scores calculated for the tool will reduce the ability to widely generalize the findings. However, the low reliability found in this study informs the need for examination and probable modification for use within this cohort, and as such, can be used as a foundation for further similar studies. Finally, an incomplete understanding of the survey questions due to English being a second language to many of the participants may have affected results.

\section{Conclusion}

Anecdotal evidence suggests that barriers to the uptake of research evidence exist for EMTs in Saudi Arabia. The limitation of barriers and the application of EBP have the potential to improve patient outcomes; however, a thorough understanding of contextual barriers is required before effective interventions can be developed. Although the BARRIERS scale requires modification for future use within a Saudi EMT cohort, this study has identified previously unidentified perceived barriers and demonstrated that similarities exist between highly rated barriers to research utilization for Saudi EMTs and other health professions. Further studies are required to resolve the complexities of research utilization within this setting. This will enable context-specific barriers to be examined and inform the development of effective strategies to increase the uptake of research evidence into Saudi EMT practice.

\section{Acknowledgment}

The authors would like to express their appreciation to the Deanship of Scientific Research at King Saud University for funding this work through Research Group Project Number RG-1438-050. We are also grateful to the Vice-Deanship of 
Postgraduate Studies and Research at the King Saud University (KSU) and the KSU Prince Sultan College of Emergency Medical Services for the support provided to the authors of this study. The authors also appreciate the contributions of Mr Raeed Alotaibi and Mr Nawaf Albaqami to this study.

\section{Disclosure}

The authors report no conflicts of interest in this work.

\section{References}

1. Claridge JA, Fabian TC. History and development of evidence-based medicine. World J Surg. 2005;29(5):547-553.

2. Balas E, Boren S. Managing clinical knowledge for health care improvement. Yearb Med Inform. 2000;(1):65-70.

3. McCluskey A. Implementing evidence into practice. In: Hoffmann T, Bennett S, Mar CD, editors. Evidence-Based Practice Across the Health Professions. 2nd ed. Sydney: Elsevier Health Sciences; 2013.

4. Grimshaw JM, Eccles MP, Lavis JN, Hill SJ, Squires JE. Knowledge translation of research findings. Implement Sci. 2012;7(1):50.

5. Guyatt G, Cairns J, Churchill D, et al. Evidence-based medicine: a new approach to teaching the practice of medicine. JAMA. 1992;268(17): 2420-2425.

6. Sackett DL, Rosenberg WM, Gray JA, Haynes RB, Richardson WS Evidence based medicine: what it is and what it isn't. BMJ. 1996; 312(7023):71-72.

7. Colquhoun H, Leeman J, Michie S, et al. Towards a common terminology: a simplified framework of interventions to promote and integrate evidence into health practices, systems, and policies. Implement Sci. 2014;9(1):781.

8. Emparanza JI, Cabello JB, Burls AJ. Does evidence-based practice improve patient outcomes? An analysis of a natural experiment in a Spanish hospital. J Eval Clin Pract. 2015;21(6):1059-1065.

9. Runciman WB, Hunt TD, Hannaford NA, et al. CareTrack: assessing the appropriateness of health care delivery in Australia. Med J Aust. 2012;197(2):100-105.

10. McGlynn EA, Asch SM, Adams J, et al. The quality of health care delivered to adults in the United States. New Engl J Med. 2003;348(26): 2635-2645.

11. Grol R, Grimshaw J. From best evidence to best practice: effective implementation of change in patients' care. Lancet. 2003;362(9391): 1225-1230.

12. Solomons NM, Spross JA. Evidence-based practice barriers and facilitators from a continuous quality improvement perspective: an integrative review. J Nurs Manag. 2011;19(1):109-120.

13. Lizarondo L, Grimmer-Somers K, Kumar S. A systematic review of the individual determinants of research evidence use in allied health. J Multidiscip Healthc. 2011;4:261-272.

14. Straus SE, Tetroe JM, Graham ID. Knowledge translation is the use of knowledge in health care decision making. J Clin Epidemiol. 2011;64(1):6-10.

15. Cone DC. Knowledge translation in the emergency medical services: a research agenda for advancing prehospital care. Acad Emerg Med. 2007;14(11):1052-1057.

16. Boström AM, Kajermo KN, Nordström G, Wallin L. Barriers to research utilization and research use among registered nurses working in the care of older people: does the BARRIERS scale discriminate between research users and non-research users on perceptions of barriers? Implement Sci. 2008;3:24.

17. Williams B, Perillo S, Brown T. What are the factors of organisational culture in health care settings that act as barriers to the implementation of evidence-based practice? A scoping review. Nurse Educ Today. 2015;35(2):e34-e41.
18. Squires JE, Estabrooks CA, Gustavsson P, Wallin L. Individual determinants of research utilization by nurses: a systematic review update. Implement Sci. 2011;6(1):1.

19. Ebben RH, Vloet LC, Verhofstad MH, Meijer S, Mintjes-de Groot JA, van Achterberg T. Adherence to guidelines and protocols in the prehospital and emergency care setting: a systematic review. Scand $J$ Trauma Resusc Emerg Med. 2013;21(1):9.

20. Metcalfe C, Lewin R, Wisher S, Perry S, Bannigan K, Moffett JK. Barriers to implementing the evidence base in four NHS therapies: dietitians, occupational therapists, physiotherapists, speech and language therapists. Physiotherapy. 2001;87(8):433-441.

21. AlShammari T, Jennings P, Williams B. Evolution of emergency medical services in Saudi Arabia. J Emerg Med Trauma Acute Care. 2017;2017(1):4.

22. Kingdom of Saudi Arabia. Vision 2030. 2016. Available from: http:// vision2030.gov.sa/ar/reports. Accessed August 7, 2017.

23. Kingdom of Saudi Arabia. National Transformation Program 2020. 2016. Available from: http://vision2030.gov.sa/sites/default/files/ NTP_En.pdf. Accessed August 7, 2017.

24. Alanazi AF. Emergency medical services in Saudi Arabia: a study on the significance of paramedics and their experiences on barriers as inhibitors of their efficiency. Int JAppl Basic Med Res. 2012;2(1):34-37.

25. Alanazi AF. Curriculum design of emergency medical services program at the College of Applied Medical Sciences, King Saud bin Abdulaziz University for Health Sciences. Adv Med Educ Pract. 2012;3:7-18.

26. Erdfelder E, Faul F, Buchner A. GPOWER: a general power analysis program. Behav Res Meth Instrum Comput. 1996;28(1):1-11.

27. Funk SG, Champagne MT, Wiese RA, Tornquist EM. BARRIERS: the barriers to research utilization scale. Appl Nurs Res. 1991;4(1):39-45.

28. Rogers EM. Diffusion of Innovations. New York: Simon and Schuster; 2010.

29. Tavakol M, Dennick R. Making sense of Cronbach's alpha. Int J Med Educ. 2011;2:53-55.

30. Kajermo KN, Boström AM, Thompson DS, Hutchinson AM, Estabrooks CA, Wallin L. The BARRIERS scale - the barriers to research utilization scale: a systematic review. Implement Sci. 2010;5(1):32.

31. Buhaid N, Lau R, O'Connor M. A survey of nurses' perceived barriers to research utilization in Bahrain in comparison to other countries. Middle East J Nurs. 2014;8(2):3-9.

32. Uysal A, Temel AB, Ardahan M, Ozkahraman S. Barriers to research utilisation among nurses in Turkey. J Clin Nurs. 2010;19(23-24): 3443-3452.

33. Sari D, Turgay AS, Genc RE, Bozkurt OD. Research activities and perceptions of barriers to research utilization among Turkish nurses. J Contin Educ Nurs. 2012;43(6):251-258; quiz 259-260.

34. Kocaman G, Seren S, Lash AA, Kurt S, Bengu N, Yurumezoglu HA. Barriers to research utilisation by staff nurses in a university hospital. J Clin Nurs. 2010;19(13-14):1908-1918.

35. Mehrdad N, Salsali M, Kazemnejad A. The spectrum of barriers to and facilitators of research utilization in Iranian nursing. J Clin Nurs. 2008;17(16):2194-2202.

36. Phillips S. Blinded by belonging: revealing the hidden curriculum. Med Educ. 2013;47(2):124-125.

37. Chen SH, Shao JH, Hsiao YC, Lee HC. Barriers to research utilization by registered nurses in Taiwan. Res Nurs Health. 2013;36(2):191-202.

38. Temel AB, Uysal A, Ardahan M, Ozkahraman S. Barriers to Research Utilization Scale: psychometric properties of the Turkish version. $J A d v$ Nurs. 2010;66(2):456-464.

39. Brown CE, Wickline MA, Ecoff L, Glaser D. Nursing practice, knowledge, attitudes and perceived barriers to evidence-based practice at an academic medical center. $J$ Adv Nurs. 2009;65(2):371-381.

40. Chau JP, Lopez V, Thompson DR. A survey of Hong Kong nurses' perceptions of barriers to and facilitators of research utilization. Res Nurs Health. 2008;31(6):640-649.

41. Hommelstad J, Ruland CM. Norwegian nurses' perceived barriers and facilitators to research use. AORN J. 2004;79(3):621-634. 
42. Omer T. Research utilization in a multicultural nursing setting in Saudi Arabia: barriers and facilitators. J Nurs Res. 2012;20(1): 66-73.

43. Buhaid N, Lau R, O'Connor M. A survey of nurses' perceived barriers to research utilization in Bahrain in comparison to other countries. Middle East J Nurs. 2014;8(2):3-9.
44. Marsh GW, Nolan M, Hopkins S. Testing the revised barriers to research utilization scale for use in the UK. Clin Effect Nurs. 2001;5(2):66-72.

45. Wallace J, Byrne C, Clarke M. Making evidence more wanted: a systematic review of facilitators to enhance the uptake of evidence from systematic reviews and meta-analyses. Int J Evid Based Healthc. 2012;10(4): $338-346$.

\section{Publish your work in this journal}

Advances in Medical Education and Practice is an international, peerreviewed, open access journal that aims to present and publish research on Medical Education covering medical, dental, nursing and allied health care professional education. The journal covers undergraduate education, postgraduate training and continuing medical education including emerging trends and innovative models linking education, research, and health care services. The manuscript management system is completely online and includes a very quick and fair peer-review system. Visit http://www.dovepress.com/testimonials.php to read real quotes from published authors.

Submit your manuscript here: http://www.dovepress.com/advances-in-medical-education-and-practice-journal 